\title{
A High Performance Multi-Structured File System Design
}

\author{
Keith Muller and Joseph Pasquale \\ Computer Systems Laboratory \\ Department of Computer Science and Engineering \\ University of California, San Diego
}

\begin{abstract}
A.bstract
File system $\mathrm{I} / \mathrm{O}$ is increasingly becoming a performance bottleneck in large distributed computer systems. This is due to the increased file $\mathrm{I} / \mathrm{O}$ demands of new applications, the inability of any single storage structure to respond to these demands, and the slow decline of disk access times (latency and seek) relative to the rapid increase in CPU speeds, memory size, and network bandwidth.

We present a multi-structured file system designed for high bandwidth I/O and fast response. Our design is based on combining disk caching with three different file storage structures, each implemented on an independent and isolated disk array. Each storage structure is designed to optimize a different set of file system access characteristics such as cache writes, directory searches, file attribute requests or large sequential reads/writes.
\end{abstract}

As part of our study, we analyze the performance of an existing file system using trace data from UNIX disk $\mathrm{I} / \mathrm{O}$-intensive workloads. Using trace driven simulations, we show how performance is improved by using separate storage structures as implemented by a multi-structured file system.

\section{Introduction}

Technology improvements in CPU speeds, memory sizes, and network bandwidths are proceeding rapidly. This progress has begun to alter the characteristics of job execution in distributed computer systems. Whereas the CPU was the performance bottleneck for job execution in the past, file I/O delays are making the file system the bottleneck. These technology improvements are also fueling the creation of new $\mathrm{I} / \mathrm{O}$-intensive applications, such as multimedia and visualization applications, which produce workload characteristics different from the commonly observed rapid cycle of creation and deletion of small files [18]. For example, ten minutes of video at 30 frames/second and 1 megabyte/frame requires 1.8 gigabytes, to be transferred at 30 megabytes/second. The large file size combined with the bandwidth requirement precludes any solution primarily dependent on increased main memory (or cache) sizes to increase performance.

Permission to copy without fee all or part of this material is granted provided that the copies are not made or distributed for direct commercial advantage, the ACM copyright notice and the title of the publication and its date appear, and notice is given that copying is by permission of the Association for Computing Machinery. To copy otherwise, or to republish, requires a fee and/or specific permission.

ه 1991 ACM 0-89791-447-3/91/0009/0056 ...\$1.50
A major impediment to improving file system performance can be attributed to the design age of current file systems, particularly with regard to their storage structures. Many of these storage structures tend to optimize storage efficiency to reduce the number of disk drives and lower overall system cost rather than focusing on minimizing disk access delays. This problem is aggravated by the difficulty of file systems to effectively divide load over their available disks. Furthermore, this will not be solved by solely focusing on making faster disks. Technology improvements related to disk drives have increased the storage density, decreased the physical size, increased transfer rates, and dramatically reduced cost, but have not significantly reduced access delays, and indications are that they will not improve in the near future.

In this paper, we describe a multi-structured file system (MFS). We expect this design to be used for large-scale file servers supporting a wide range of intensive workloads. It addresses the requirements of applications with very high I/O requirements, such as databases supporting large objects, multimedia applications which include video and audio, and scientific applications including visualization, executing on computers ranging from high-performance workstations to general-purpose supercomputers.

Our design is based on combining the positive attributes of several well known techniques, such as caching, disk striping, disk mirroring, using append-only log storage, and using disk arrays, to achieve performance improvements which increase with the number of disks used. In Section 2 we describe past work. In Section 3 we examine the characteristics of a typical file system workload in terms of these modes of disk access. In Section 4 we describe a design for MFS. In Section 5 we present a trace driven simulation study comparing an existing Berkeley Unix Fast File System (FFS) [13], and MFS. We present our conclusions in Section 6.

\section{Past Work}

Disk striping is a technique for improving throughput by dividing a logical disk storage unit evenly across multiple disks such that whenever that storage unit is requested, concurrent transfers of blocks from each of the disks occurs. However, average access times can be large in disk striping systems since access time is governed by the disk with the largest access time. Garcia-Molina [7] proposed a solution based on immediate reading which allows a disk read to occur anywhere within a block, and this was found to improve performance considerably. Kim [10] developed a solution to 
this problem based on hardware synchronization of the disks.

Disk striping has difficulty in supporting concurrent access I/O: although bandwidth can be increased for large data requests, the disks cannot be used in an independent manner to service many small independent concurrent file requests. Livny [12] conducted a study of clustered storage, where logically related data blocks are clustered on a single large disk, and declustered storage, where logically related data blocks are spread over multiple disks, as in disk striping. Declustering performed better than clustering for most workloads; however, under high loads with uniform access patterns, clustering worked better. Furthermore, as the load increases, declustered storage shows a noticeable increase in the number of seeks and length of seek delays. Reddy [22] compared the performance of combinations of disk synchronization and data declustering/disk striping techniques. Configurations which consisted of only synchronized disks performed well at low request rates, while increasing the amount of declustering improves performance at high request rates. These studies all demonstrate the sensitivity of striped storage to request rates.

Disk arrays are systems of multiple disks arranged to provide higher $\mathrm{I} / \mathrm{O}$ bandwidth than a single disk of aggregate size of the disk array. There is the problem of reliability when file information is spread across multiple disks: a failure in any disk makes (at least part of) the file inaccessible. A simple improvement is through redundancy using mirrored disks, as described by Britton and Gray [1]. This also reduces access time for concurrent reads, although writing incurs additional delays. Patterson [20] showed that large arrays of lower performance disks can have better performance, lower cost and improved reliability over a lesser number of more expensive disks. Error correction blocks are spread over all the disks so that single disk failures could be handled, and no single disk has to be devoted to ECC, which would be a potential bottleneck.

Disk block caching [25] also improves file system performance by keeping recently used data blocks in memory to service future requests without incurring disk delays. Ousterhout [18] studied dynamic access methods of a Unix file system, and found that using a very large cache leads to a drastic reduction in disk I/O traffic since, in the workloads observed, most files are small and most are short lived. Satyanarayanan [24] made similar observations of file sizes and lifetimes by studying static snapshots of a TOPS-10 system. Nelson [16][15] studied the effects of combining local and remote caching in a diskless workstation environment with a central file server, again noting the benefits of a very large cache at the file server, but reducing server traffic by local caching.

Logging is a technique where data is always written to a new unused area of storage, thus old data is never overwritten. With the advent of WORM optical disk drives, log structured storage has become attractive for designing reliable file systems with version support, as demonstrated by the early work of Svobodova [26], and more recently by Gait [6] and a number of others. Hagmann [8] designed a log structured file system for a workstation programming environment. Cheriton [2] describes a file system extension to implement $\log$ files on WORM drives.

Log structured storage has the interesting property that the next write will always start at the position where the last write was finished. For workloads which allow most file reads to be serviced by an in-memory cache, a log structured storage can effectively be used as the permanent storage of a file system. Ousterhout [19] and Rosenblum [23] describe a circular log structured file system that is optimized for writes, and works well for office environment workloads which involve accessing mostly small files.

However, performance problems may arise for workloads where the working set of file data is difficult to keep in the cache. When read requests must go to log store, the disk head must move between the current write point and the location of the file on log storage. Under workloads with concurrent read and write requests this can become very expensive. Such workloads are not unusual, and may become more prominent with time. Examples include workloads exhibiting sequential reading of very large files (e.g., a file containing a library of fonts, or a video file which can easily fill up any cache), or shell commands which touch a large number of files (e.g., a string search command like the Unix grep, recursively applied to a large file system tree). For workloads where these type of requests are made at a high rate, even the largest of caches can be easily defeated.

Other relevant file system studies and/or techniques for performance improvement include the overhead of looking up file names in directories by Floyd [4], the value of contiguous storage allocation for files by Tanenbaum [27] and Yokoyama [28], and the value of storing file data in file headers by Mullender [14].

\section{Characteristics of File System Workloads}

To understand file system operation under actual operating conditions, we extensively instrumented the file system of a 4.3BSD-Tahoe kemel running on a Unisys $7000 / 51$ under a real workload. The hardware consisted of 64 megabytes of main memory, 64 user ports, ethernet, and three disk controllers, each controlling a single Fujitsu 2361A (Super Eagle). The buffer cache size was 7 megabytes, and all file systems consisted of 8 Kbyte primary blocks with 1 Kbyte fragments (see [13] for a description of FFS).

The file system was measured over periods characterized by fairly high workloads. There were a total of nine 75-minute measurement periods, with system activity characterized by the following statistics. The average number of users was 60 , about $75 \%$ of these users were performing edit-compile-test cycles to build the code generator for a compiler, and the remaining users were performing database operations on large disk files. The run queue contained an average of 9 to 15 active jobs with close to $100 \%$ CPU utilization. The virtual memory sys- 
tem had between 30 to 50 megabytes free at all times. There were no pageouts, process swaps, or page scans. The file system was finely tuned, with load being well balanced between the three disks.

We obtained trace data by placing checkpoints [3] at the entry and exit points of every system call that might potentially cause any file system operation, and within all major routines which affect file system-related data structures. Each trace event was timestamped by a hardware clock providing a resolution of 2 milliseconds. This allowed us to determine the execution flow from any system call entry point to its destination, including any cache or any disk; determine every internal file system event (such as a disk request or cache miss) generated by a specific file system request; and determine the service rates of all major internal file system functions (e.g., name lookups, buffer cache lookups, disk operations). Figure 1 illustrates the location of the checkpoints.

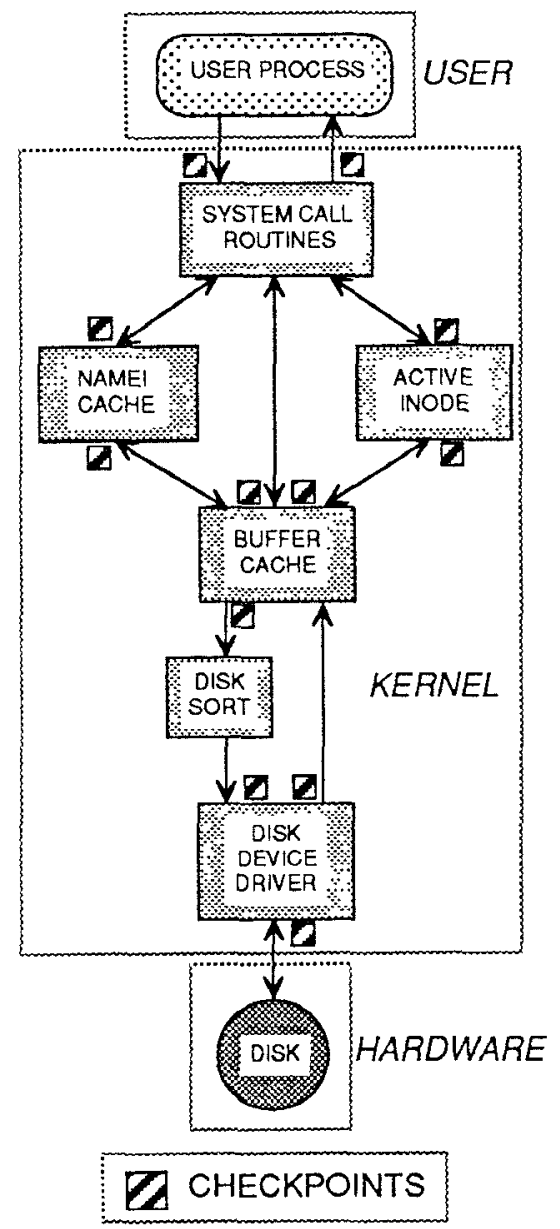

Figure 1. Location of File System Checkpoints

To reduce measurement interference and not affect local disks, trace data was transmitted over a network and logged on a remote host. The total CPU overhead due to all measurement activity (including transmission overhead which was minimal) was under $5.0 \%$ of measured capa- city.

We distinguish two types of file requests: data and control. Data requests mainly operate on data storage, which is that part of the file system storage containing the actual contents of files. The Unix read and write system calls correspond to data requests. Control requests operate on control storage, which is that part of the file system storage containing access control and other descriptive information about files. Requests for file creation, deletion, renaming, truncation, and identification are examples of control requests.

Table 1 shows statistics for events generated by read, write, and control storage requests (which include open, close, unlink, sync, access, chdir, chmod, chown, dup2, exit, fsync, ftrunc, mkdir, link, rename, rlink, rmdir, stat, utime) which make up the remaining file systemrelated system calls. All statistics are averages taken over all the trace periods.

Data requests account for $39 \%$ of all file system requests, while the remaining $61 \%$ are due to control requests. Furthermore, read system calls dominate write system calls by a ratio of 3.2 to 1 (this agrees with measurements in [18]). Data requests cause $36 \%$ of all disk operations, and of these, disk writes dominate disk reads by a ratio of 1.6 to 1 . When control requests are included, this ratio increases to 3.6 to 1 . When considering only control requests, disk writes dominate disk reads by a ratio of 7.1 to 1 .

\begin{tabular}{|l|c|c|c|r|}
\hline \multicolumn{5}{|c|}{ System Call Events Per Period } \\
\hline $\begin{array}{c}\text { System } \\
\text { Call }\end{array}$ & $\begin{array}{c}\text { Number } \\
\text { Requests }\end{array}$ & $\begin{array}{c}\text { Total } \\
\text { Disk }\end{array}$ & $\begin{array}{c}\text { Disk } \\
\text { Read }\end{array}$ & $\begin{array}{c}\text { Disk } \\
\text { Write }\end{array}$ \\
\hline read & 55475 & 6424 & 5368 & 1056 \\
\hline write & 17413 & 9062 & 499 & 8563 \\
\hline control & 116215 & 27377 & 3379 & 23998 \\
\hline Totals & 189103 & 42863 & 9246 & 33617 \\
\hline
\end{tabular}

Table 1

Caching is a major influence in converting read dominated system call requests to write dominated disk requests, as was observed in [16]. Upon measuring the hit rates of various caches, we found that the disk cache had a $87 \%$ hit rate, the name lookup cache had a $83 \%$ hit rate, and the inode cache (system inode table) had a $63 \%$ hit rate.

Consider the relationship of read and write system calls to read and write disk requests. $58 \%$ of disk reads are due to read system calls, while only $25 \%$ of disk writes are due to write system calls. Furthermore, while the rate of disk reads due to read system calls is slightly greater than the rate of disk reads due to control requests, the rate of disk writes due to control requests is much greater than the rate of disk writes due to write system calls.

Finally, we considered the completion time and the data request size for data and control system calls. These statistics are summarized in Table 2. All statistics are per 
period, averaged over all nine periods.

The completion times for control system calls had a wide variation, from 10 milliseconds to 303 milliseconds, with an average value of 27 milliseconds, which is almost double the average completion times of nearly 15 milliseconds for read and write system calls. The main reason for this is that control requests access the disk more often than data requests. However, note that the average amount of data requested by control system calls is almost 20 times smaller than that of read or write system calls. Given that the average disk request time was $23 \mathrm{mil}-$ liseconds, the memory caching of control information and data was, to differing degrees, effective for both control and data requests.

\begin{tabular}{|l|c|c|}
\hline \multicolumn{3}{|c|}{$\begin{array}{c}\text { System Call Completion Time } \\
\text { and Request Size Per Period }\end{array}$} \\
\hline System Call & Time (msec) & Size (bytes) \\
\hline read & 16 & 4235 \\
\hline write & 14 & 4688 \\
\hline control & $10-303$ & 252 \\
\hline
\end{tabular}

Table 2

In Figure 2, we show the arrival of disk requests broken down by data reads, data writes, control reads and control writes. Notice how control writes are continuous and control reads are clustered. The frequency of control requests is so high that it is difficult to maintain disk positioning locality for data reads and writes.

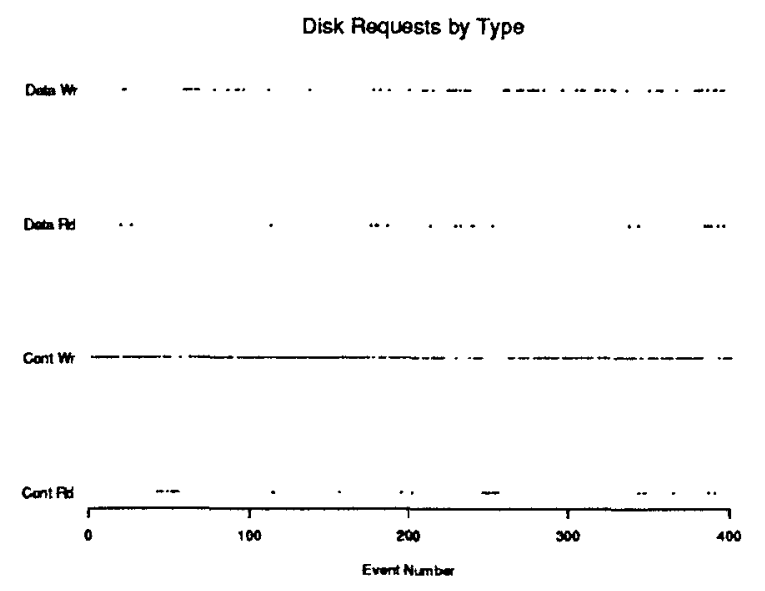

Figure 2. Disk Requests by Type

Our observations indicate that control storage has very different access characteristics than data storage. They also suggest the importance of considering the requirements of control storage operation for improving file system performance, and whether a single storage structure can effectively respond to both data and control requests. Upon analyzing our trace data in the time domain, we noticed the degree to which small control requests mixed with much larger data requests (generally stored at different locations on disk) conflicted. These conflicts are due to induced disk head seeks, and result in increased service times for both control and data requests.

We emphasize that the conflict between control and data requests is a general problem; our trace data allowed us to observe this problem, however, we believe these observations would appear on any file system where control and data is mixed (which includes the vast majority of file systems in existence today). We expect the trend towards larger data files with larger average data request sizes to continue. As the size of data requests increase, the conflict between control and data request characteristics also increases. This trend provides further motivation to develop techniques which address the requirements of both control and data storage for improving file system performance.

\section{The Design}

Our approach is based on a suggestion by Powell in a study of the DEMOS file system [21], where one tries to understand properties of the file request distributions and then develop solutions to take advantage of those properties. We looked for properties in request patterns of system calls and disk accesses which we expect are present in a wide range of file system workloads. Our observations led to the following two design principles:

- Separate control requests from data requests. Control requests are typically much smaller than data requests, but they occur more often. Separate storage structures for control and data are provided, each designed to perform best under their respective request-pattem characteristics. This also provides a natural division of load across parallel storage structures.

- Separate cache backups from cache flushes. We distinguish between a backup request that writes cached information to non-volatile storage to guard against information loss due to crashes, and a flush request that writes cached information, which is not expected to be used soon, to non-volatile storage to make room for other information. To insure the highest level of reliability, backup requests must be done immediately upon file system modification. This leaves flush requests to be made at more convenient times as determined by the file system. Based on the different timing characteristics of these write requests, separate optimized storage structures are provided.

\subsection{Design Overview}

MFS contains three non-volatile storage structures: control, data, and log storage. In addition, three caches, two for control storage and one for data storage, are used to improve read performance. Figure 3 illustrates the basic relationships of these major components. 
Control storage and data storage provide long term storage for information that has not been recently modified. The caches store recently-accessed information. They are filled from control or data storage as appropriate, and they are eventually flushed to a corresponding long-term storage structure if modified. Log storage is used purely for cache backup (based on a write-through policy) to guard against information loss during a crash. During normal system operation, only append-writes are issued to log storage.

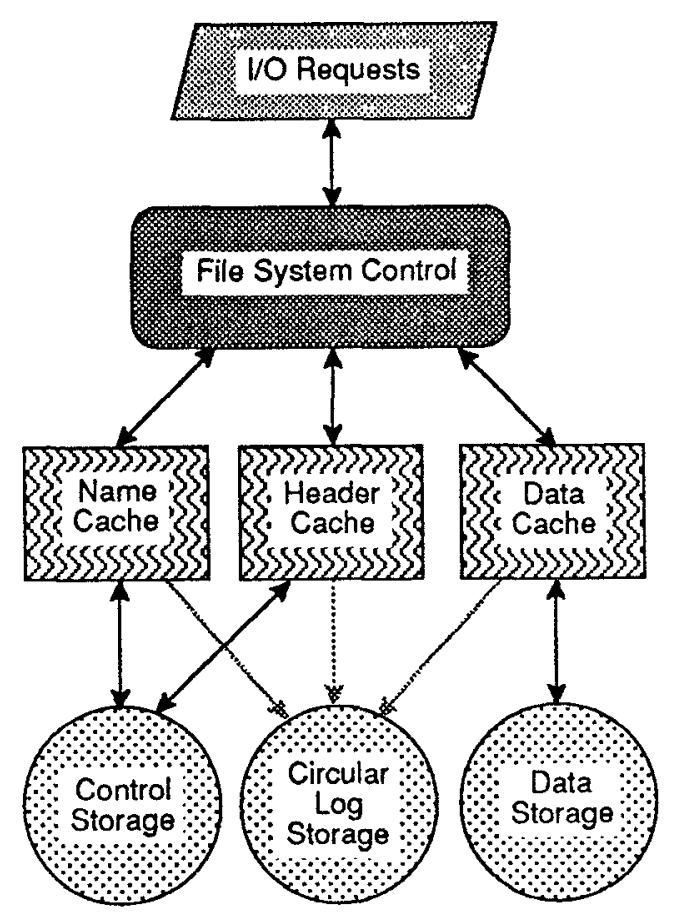

Figure 3. Multi-Structured File System

\subsection{Control storage}

Control storage is the long term storage for file control information, which includes file attributes, storage maps, and directory information. Control information is organized in two distinct tables, the file header table and the name map table. A file header consists of an attribute record, which describes the attributes of a file such as ownership, access protection, access times, etc., and a storage map, which describes how to locate the file's data on data storage.

The storage map is an extent-based table. Each entry contains a starting block address and a length. Data storage is allocated in units of striped blocks or striped tracks, depending on the request size. If the file data size is smaller than the entire storage map size, then the file data is stored directly in the file header replacing the storage map implementing an immediate file [14]. This prevents small file data requests from having to be served by data storage and improves performance for small files. Block size, striping factor, and storage map size are tunable parameters.
The name map table binds file names to files and organizes file names into directories. All naming information is isolated from file data (unlike many file systems which store the name information as special file data). Each file header is referenced by one or more entries in the name map table, allowing a file to have more than one name. The name map table is organized as a chained tree structure similar to Yokoyama [28] to cluster directory information.

Control storage is physically allocated as follows. For either the file header table or the name map table, disk storage is allocated by cylinder. The first blocks in each cylinder contain configuration data followed by a bit map that describes storage for that cylinder. The configuration data contains the number of disks and disk geometry data (e.g., tracks, blocks per track, etc). The bit map is used to locate those blocks that contain at least one free entry (file header or name map). Following the allocation map, the name map or file header tables are allocated in consecutive blocks, then track by track for the entire cylinder.

The characteristic access pattern of control storage is that of many concurrent small read/modify/write cycles; this suggests the effective use of an array of very small disks to contain control storage. (Although the size of control storage is typically less than ten percent of data storage [18], for the very large file systems warranting our multi-structured design, the absolute size of control storage can require one or more disks). The regular structure of the tables described above allows control storage performance to be scaled through the use of a modified version of disk mirroring techniques [1][20]. This improves concurrent access and reduces the queueing delays that tend to dominate control storage requests. Since many disks are capable of handling a request, load is easily divided among the mirrored disks. The control cache keeps track of which disks have been updated and does not flush a modified entry until all disks have been updated. (Log storage will alway have a valid copy, so a crash while the mirrored disks are out of sync is recoverable). This technique avoids single threaded writes to mirrored disks and extends concurrent operations to both reads and writes.

\subsection{Data Storage}

Data storage is the long term storage for all file data (except those files that are small enough to be stored as immediate files). Data storage is structured using disks configured for striped access to optimize for high bandwidth sequential I/O. Allocation is simply in units of striped blocks and striped tracks, which contain the same positioned blocks and tracks on each disk drive, and is based on methods similar to those used for contiguous files [27][28].

The first track(s) on each disk contains configuration data and the allocation bitmap for data storage. The configuration data contains information about drive geometries and the number of drives. The allocation bitmap is structured as a two-level tree, the lowest level corresponding to striped blocks, and the 
second level corresponding to striped tracks. A striped track entry is marked free only if all the striped blocks it comprises are marked free. To find a free striped block (or blocks), or a striped track, the corresponding level of the tree is examined. This two level bit map is similar in concept to that used in [28]. Figure 4 illustrates the layout of a data allocation map.

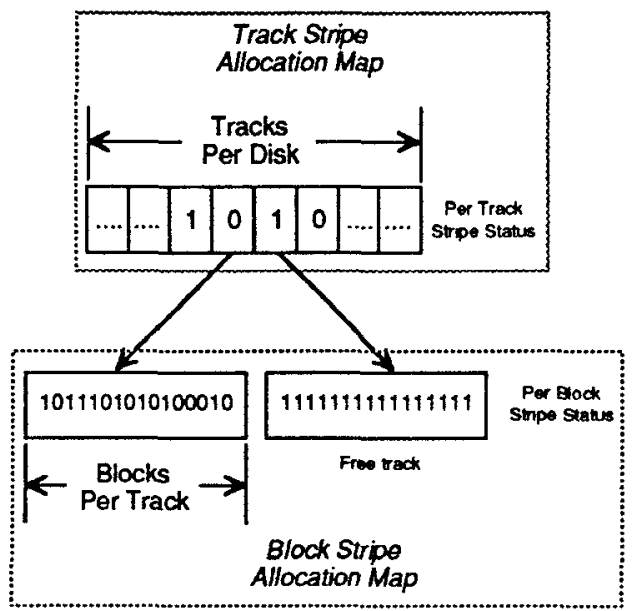

Figure 4. Data Allocation Map

\subsection{Cache Operation}

Cache entries are filled with data read from either control or data storage as appropriate. A LRU (least recently used) replacement policy combined with a writethrough update strategy [25] to log storage as the backing store are used. A cache flush operation writes dirty entries to either control or data storage as appropriate, by writing the current version from cache memory.

To improve performance, a hash table (with chaining) and three lists (LRU, free, and locked) are used to organize the entries. The LRU list orders valid entries from most-recently accessed to least-recently accessed. The free list contains flushed entries (but may still contain valid data). The locked list contains entries which should remain locked in the cache, such as those currently involved in an 10 operation, or those containing file headers for currently open files.

Each cache consists of a table of entries whose content varies by the type of cache. Each cache entry contains valid and dirty flags, the location on disk storage where this entry is located, a pointer to an in-memory copy of the storage, pointers to support the hash chain, the LRU, free, and locked lists, and the log storage chain (described in Section 4.5). A data cache entry includes a pointer to a memory copy of data storage, which may range in size from a striped block to a striped track, and is hashed by storage location. The file header cache is hashed by file header table index. A file header cache entry includes a reference count, a pointer to a memory copy of the file header and a pointer to data cache memory for large I/O operations (described below). Two hash tables provide fast access to the name map cache entries, one providing access by name map table index, and the other by path name (similar to what exists in FFS [13]). A name map cache entry includes a pointer to a memory copy of a name map block and a reference count of path cache entries that point at it.

The system tries to keep a minimum number of entries on the free list for fast allocation whenever a cache miss occurs. This is implemented using a watermarkbased strategy to limit the number of dirty entries (a dirty entry has a copy of data that is a newer version of what is contained in data or control storage). Associated with each cache is a high and a low watermark. In normal operating mode, the cache does not flush dirty entries.

When the number of dirty cache entries reaches the high watermark, the cache goes into a flushing mode. While in flushing mode, whenever a file system request generates a cache hit, a cache flush operation is also started for the oldest dirty cache entry. The effect we wished to achieve was that of spreading flush operations over time to amortize their cost, and overlapping cache flush operations with file requests which are successfully serviced by the same cache. Cache hits are used as a heuristic to indicate low expected traffic to the corresponding non-volatile storage structure. The cache remains in flushing mode until the number of dirty entries is reduced to the low watermark level.

Certain file system requests pattems may cause degenerate behavior in the file system. The primary problem addressed by our design concerns sequential $y / O$ on large files. Sequential YOO on large files can quickly flush the contents of the data cache and degrade overall performance. (This is a problem with all file systems that use caches, not just our design). To reduce this effect, a pointer is maintained in the file header cache. As the result of sequential $\mathrm{YO}$ requests on a large file, caching for this file is limited to a single entry in the data cache. (The size of the memory storage referenced by this single entry is the size of a large I/O request, which can be at most a striped track). The pointer in the file header cache is set to the address of this entry. This causes all subsequent data $\mathrm{I} / \mathrm{O}$ operations on that file to reuse the single cache entry. For large write operations (e.g., the entire striped track), the cache entry is flushed immediately to data storage and not written to log storage. Although the cache entry is valid, it is never marked as dirty. If the pattern of data access changes, the pointer is cleared and normal operation is resumed.

\subsection{Log Storage}

Log storage backs up all caches. It is designed to optimize writes and is never read during normal operation. (However, during crash recovery operations which precede normal operation, it would be read to update control and data storage). When the file system begins normal operation, log storage is empty. Whenever a cache entry is written, the log storage is updated. Regardless of the kind of data involved (control data or file data), all updates are appended to the log storage. 
Log storage updates are either cache backup records, or cache fush records. A cache backup record consists of a header block followed by one or more data blocks. The header block describes the permanent location of these data blocks on either control or data storage. A cache flush record consists only of a header block, and is written after a cache flush operation completes to record the time at which a specific data or control storage location was made consistent with the copies in a cache (which happen to also be recorded on log storage). This is required for crash recovery as data and control storage blocks do not have time stamp fields.

Log storage is organized in a circular structure such that the oldest written area of storage is reclaimed for a new record to be appended. Any data block in the log storage can be characterized as follows: dirty, the only other copy exists in one of the caches; flushed, an up-todate copy exists in control or data storage; or invalid, a newer version exists in control, data or log storage. For reclamation, only dirty blocks need to be considered since they are not saved anywhere but the caches. (However, since dirty blocks are contained in the caches, no read operations on log storage are ever required for reclamation).

A data structure called the log storage chain keeps track of locations of dirty data blocks, and each entry is double linked to the corresponding cache table entry. Entries in the log storage chain are ordered according to the order in which dirty data blocks are written, (which also occur in the same order on log storage). The entry at the head of the chain is the dirty data block closest to the current log write point, while the end of the chain is farthest away. Whenever a cache entry is first modified, a new entry is added to the log storage chain. Whenever a cache entry is flushed, the corresponding entry in the log storage chain is simply removed.

To efficiently implement storage compaction (again, without having to read from log storage), the system maintains two pointers to blocks in log storage: the write point (the current end of the $\log$ ), and the examine point. A cache write to log storage moves the write point and the examine point by the number of appended blocks. A new entry is then added to the end of the log storage chain to describe this newly written data. If the examine point has moved past the storage location of the first entry on the log storage chain, the first entry is rewritten at the write point (nothing is ever actually read or written from log storage at the examine point). The log storage chain entry describes the cache entry where the data can be obtained to be written to log storage (so no read operation from log storage is needed). This data is rewritten by appending it to log storage, the write and examine points are updated by the number of blocks written, and the log storage chain entry is updated and moved to the end of the chain (it is now the furthest away from the current write point). The examine point is again checked against the first entry to see if another entry needs to be moved. If so, the operation of compacting storage is repeated. Figure 5 illustrates a log storage chain and a portion of log storage.
The size of log storage should be made large enough so that the majority of log storage will be reclaimable without performing a compact operation, in order to avoid extended periods of continuous log storage movement. This is achieved if log storage size is at least twice the size of total cache storage; in practice, this ratio would be tailored to the workload.

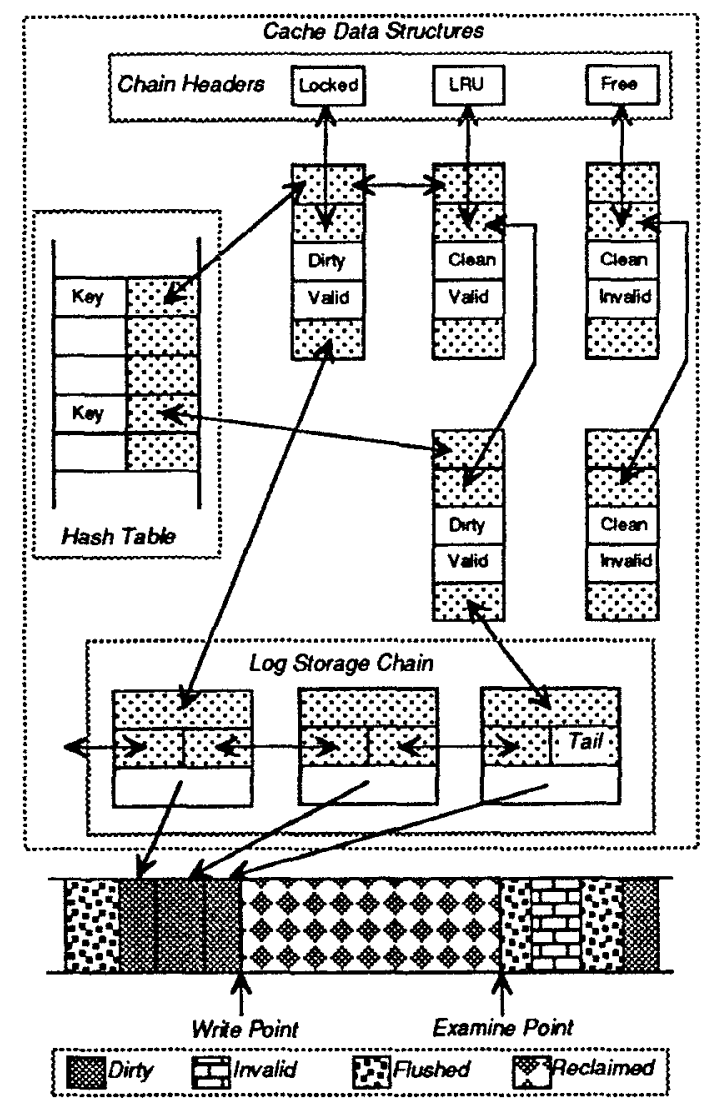

Figure 5. Log Storage Structures

Log storage is allocated as follows. Assume for the moment that there is only one disk with a single two-sided platter. Tracks are allocated sequentially from outermost to innermost, using only the top face of the platter. When all these tracks are allocated, then the tracks on the bottom face are allocated sequentially, this time starting from the innermost to outermost. This closes the loop, creating a circular structure where the next track is always one disk seek away.

If multiple disks with separate controllers are available, disk head prepositioning can be done in such a way that seek and latency delays can be minimized. The general allocation algorithm (assuming two-sided platters) is as follows.

Given D disks (with separate controllers), P platters/disk, and $T$ tracks/platter, where tracks are numbered 1 to $T / 2$ on the top side physically ordered outermost to innermost, continuing with $(T / 2)+1$ to $T$ on the bottom side physically ordered innermost to outermost: 
do forever

$$
\begin{aligned}
& \text { for } t=1 \text { to } T \\
& \text { for } p=1 \text { to } P \\
& \text { for } d=1 \text { to } D \\
& \text { allocate track }(d, p, t)
\end{aligned}
$$

Figure 6 illustrates the physical allocation of a $\log$ storage with two disks and two platters. The numbers show the order in which the first eight tracks are allocated.
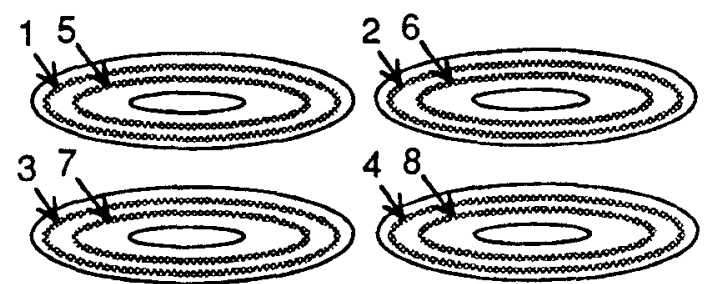

Figure 6. Physical Allocation of Log Storage

After a track has been written, a seek operation is immediately issued for that disk to preposition the disk head. However, a write to that disk will not occur until a track on each of the other disks have been written. Consequently, if $(D-1) t_{\text {trensfer }}>t_{\text {seeck }}+t_{\text {transfer }}$, where $t_{\text {transfer }}$ is the time to transfer a track of data to disk, and $t_{\text {seek }}$ is the time to seek to an adjacent track, the disk head will always be prepositioned correctly for the next write to that same disk. Furthermore, if disk controllers are equipped with track buffers, rotational delays are minimized. The net result is that cache backup operations incur minimal disk access delays and can complete asynchronously.

In summary, the advantage of log storage is that the only operation it must support is append-writes. Since the size and allocation order of storage within the disk loop is predetermined, the exact location of the next write is always known. If the number of disks in the loop is large enough, both a data transfer and a seek operation can be completed on a disk before the next write operation to that disk is issued.

\section{Performance}

To verify our claims, we conducted a performance evaluation study using a trace driven simulation of our file system design. Our primary goal was to verify that separating storage into the three isolated areas (control, data, and $\log$ ) would significantly improve performance.

We constructed two simulators, one of the existing FFS to which we had access, and one of MFS. Each simulator was driven by system call-level trace data. Most of the traces we used were generated from real workloads (described in Section 3) to validate the FFS simulator and to compare the performance of the two file systems. Others were generated from artificial workloads we constructed for controlled experiments designed to test the performance limits of MFS.

The simulators are quite detailed. Most of the inmemory data structures are real, such as caches with LRU block replacement (in fact, much of the FFS simulator executed the actual FFS kernel code). The disk hardware is simulated such that each disk's access time depends on seek and transfer time based on the current head position for that disk and the size and location of the next request. Mechanical parameters for the all disks such as rotational speed and seek time as a function of distance are based on a Fujitsu 2361a [5]. We used disks with the same accessperformance characteristics in both simulations to eliminate the effects of different hardware performance in our studies.

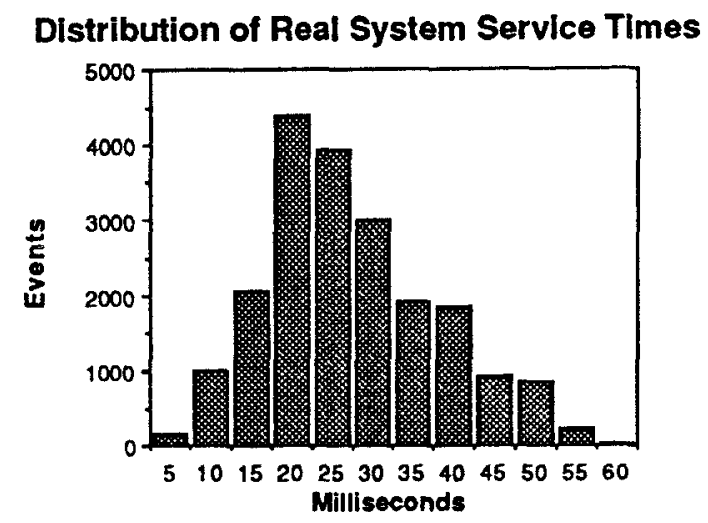

Distribution of Simulated Service Times

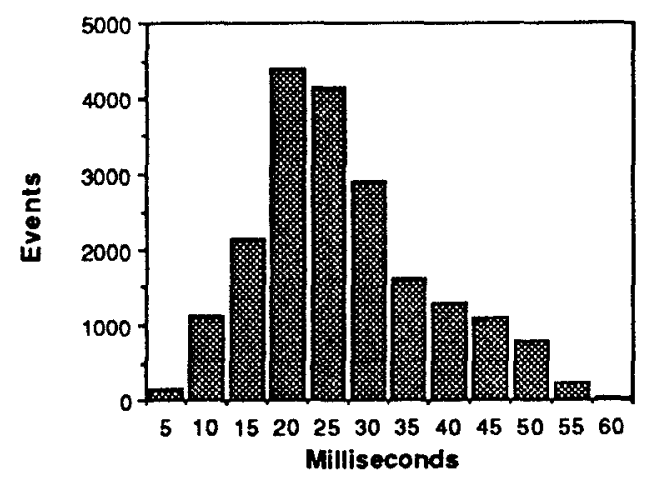

Figure 7. Validation Results

We validated our model of FFS by emitting service times it calculated for each individual system call and for all disk level accesses, and compared these to the measured system call and disk access service times by comparing the respective distributions. In almost all cases, the mean squared error between the distributions was under $5 \%$. Figure 7 shows a distribution of service times for access of the disk (which is the main simulated object) measured in the actual system, and the distribution produced by the simulator.

\subsection{Ability to Handle Increasing Loads}

In these experiments, we compare the ability of the two file systems to handle file loads of increasing intensity. For MFS, we used one small disk for control storage, one large disk for data storage, and two small disks for log storage. The total disk space for MFS and FFS were effectively the same (although the space was organized differently with a different number of disks and disk controllers). The file systems also had the same effective cache sizes. 
Seven experiments were carried out, each with a progressively more intensive load. Loads were constructed by combining multiple sets of different traces so that requests are issued concurrently. All the traces individually generated a load of approximately the same level of intensity. Thus, the load produced by a single trace is taken to be a unit of intensity of load, and a load of $n$ signifies the composition of $n$ traces read in parallel.

Figure 8 illustrates the higher rate of performance degradation with increasing load for FFS relative to MFS.

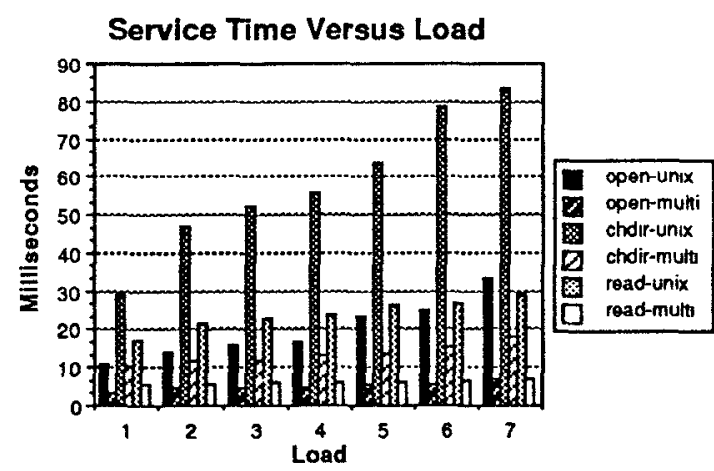

Aggregate Service Time Versus Load

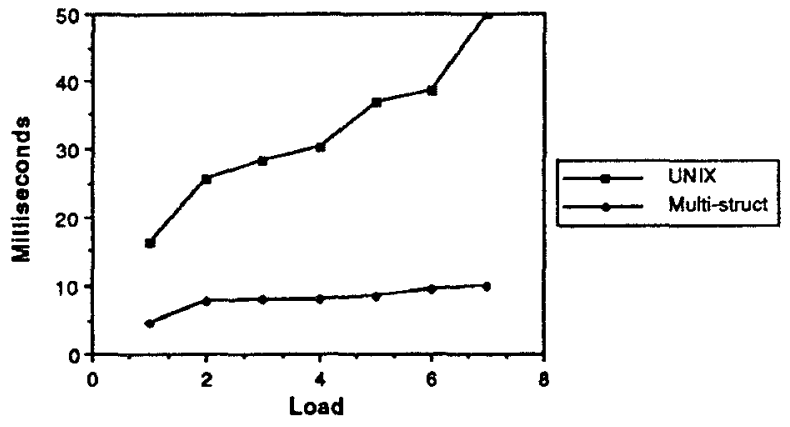

Figure 8. Comparison of Service Time Versus Load

MFS is less sensitive to increased load than FFS, and it exhibits a significantly better performance decay rate.

\subsection{Ability to Handle Large $1 / O$}

We now examine the performance of the two file systems when handling large data $\mathrm{I} / \mathrm{O}$ requests for reading and writing. The purpose of these experiments is to measure the best large I/O performance each file system is capable of providing under optimal conditions. Trace data was obtained by tracing our real FFS with an artificial execution environment of programs we constructed which continually make large file $1 / O$ requests.

We conducted four experiments; in each one, 50 Mbytes of data was read from or written to a file. In the first experiment, 50 Mbytes were written sequentially to a file in 1 Mbyte increments. The second experiment is the same, except that data is read. In the third experiment, 50 Mbytes were read in randomly ordered 1 Mbyte requests (however, nothing is read more than once). The fourth experiment is similar, except that data is written. Figure 9 contains the results. For MFS plots, performance is shown for different data storage sizes (i.e., number of striped disks).

The significant differences in performance are due to the different techniques used by the two file system to allocate and reference data storage. Under FFS, the file map (inode) points to blocks that are no larger than 8 Kbytes each, while the file map in MFS supports pointers to striped blocks and striped tracks.

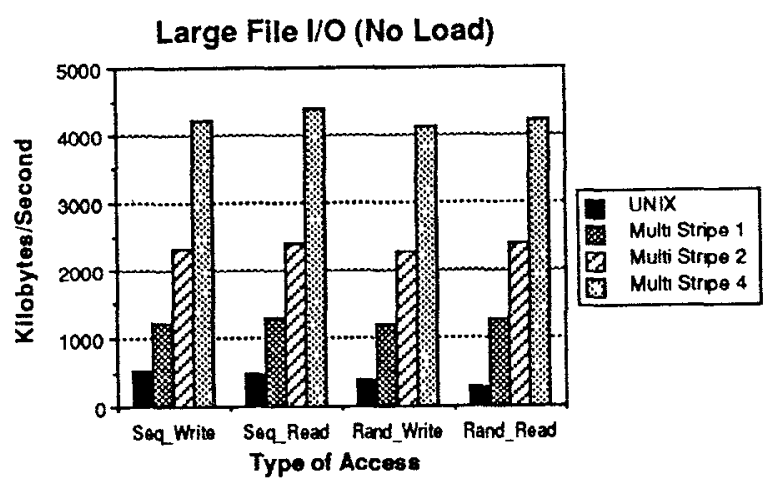

Figure 9. Large I/O Performance

\subsection{Large I/O with Competing Load}

We conducted three experiments where a 1 Mbyte data request is made which competes with no load, one unit of load, and two units of load. The first experiment with no load tells us the maximum throughput for each file system by transferring 1 Mbyte of data as fast as possible, which was $500 \mathrm{~Kb} / \mathrm{sec}$ for FFS, and $1300 \mathrm{~Kb} / \mathrm{sec}$ for an equally configured MFS. Although the latter's performance is much better, we are more interested in the rate of performance degradation of each file system when competing load is added, which is the purpose of the second and third experiments. Figure 10 shows the 1 Mbyte read throughput for each file system, expressed as a percentage of the maximum possible throughput for that file system.

1 Mbyte Read With Competing Load

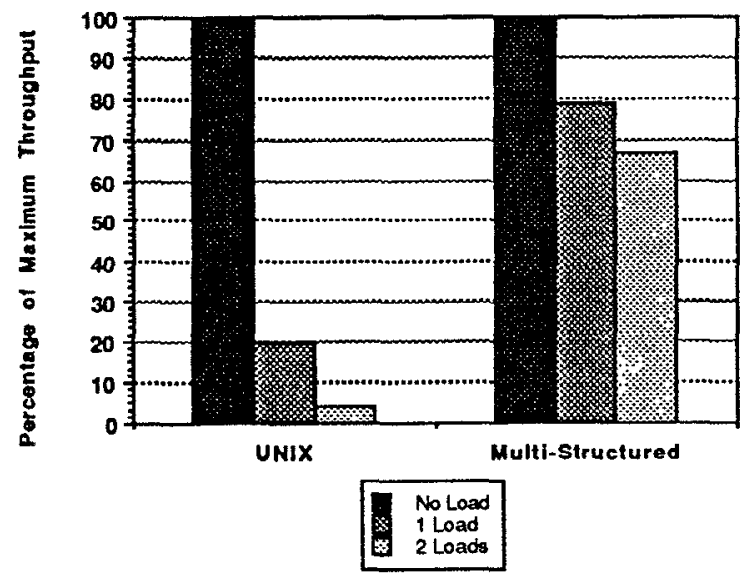

Figure 10. Large $1 / O$ with Competing Load 

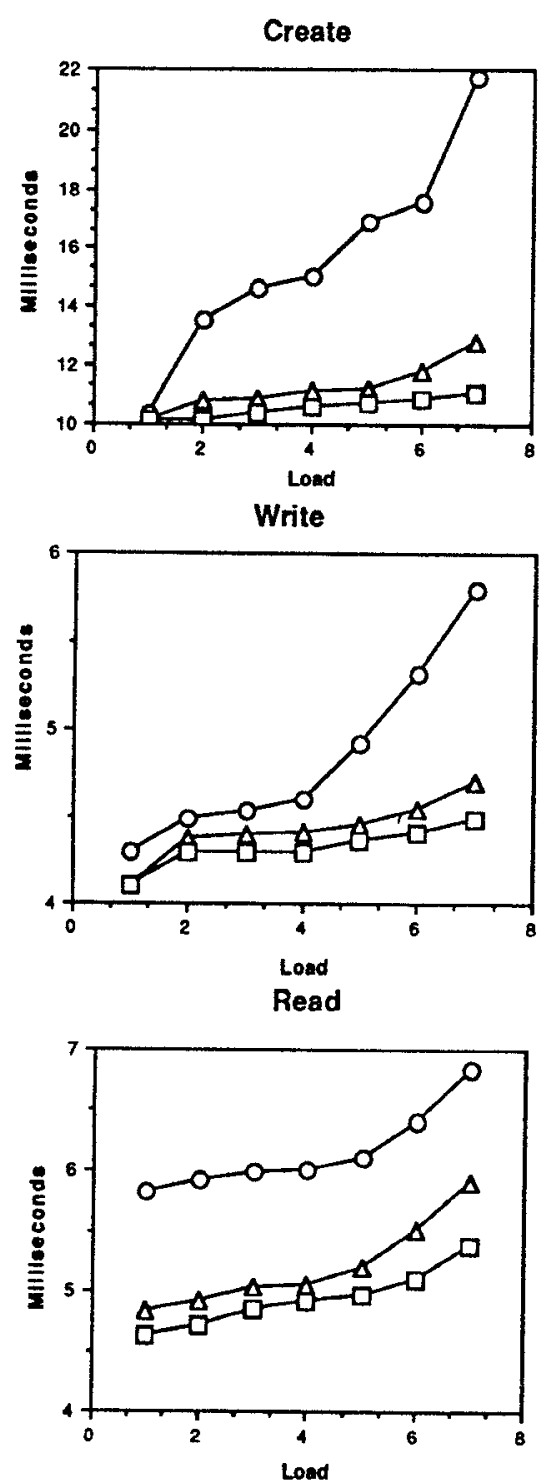

Cache Flush
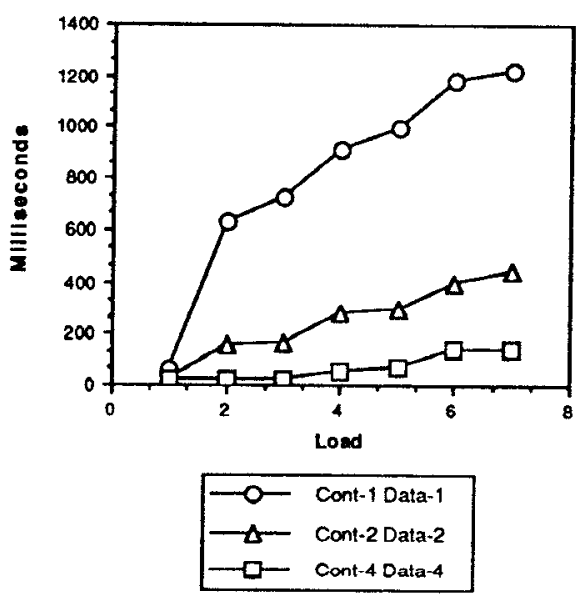

Figure 11. Performance Scaling Effects
For FFS, the 1 Mbyte read rate quickly declines to $20 \%$ of the maximum throughput with 1 load, and to less than 5\% with 2 loads. For MFS, the performance degradation is noticeably less. The source of performance degradation here is due to the conflicts between the many concurrent small control requests of the competing load with the single large 1 Mbyte data request that severely limits the performance of FFS. MFS performs well because it separates control storage from data storage, because of their characteristically different access patterns.

\subsection{Performance Scaling}

In this experiment, we examine the performance of MFS under different disk storage configurations while varying the number of concurrent request loads from 1 to 7. The goal of this experiment is to verify the ability of the file system to increase performance to offset increases in the request load. In Figure 11 we plot performance of various system calls as we vary the size of control and data storage structures concurrently from 1 to 4 disks each. In each graph, different curves are plotted for each disk configuration. These curves are labeled by the number of disks in control and data storage. For example Cont-2 Data-2 means 2 mirrored disks in control store and 2 striped disks in data store.

A noticeable improvement is obtained by increasing the storage size. In fact, with 4 disks in control storage and 4 in data storage, under 7 concurrent loads, the service times for all system calls are better than for 1 disk in each of data and control storage structure with only a single load.

The lower plot in Figure 11 shows the service time for cache flush operations. Cache flushing requires writing dirty blocks to either control or data storage, and is a very good indicator of the level of workload those two storage areas are servicing. The service time is noticeably high for the smallest configuration of one disk in each of control and data storage. When these two storage structures are increased in size, the service times for cache flush operations are significantly reduced.

These experiments have illustrated the ability of MFS to scale performance by simply adding additional disk drives to match workload requirements.

\subsection{Discussion}

Under MFS, control and data storage are located on different disks, while on more traditional file systems each disk is divided into distinct areas for control and data storage (e.g., cylinder groups in FFS). Separating the location of control and data on different disks versus storing both control and data on every disk does not create any additional software complexity, as the same type of data structures are used. In face, this division results in a more uniform usage of disk storage which simplifies the allocation of disk storage.

This simple separation of control and data leads to performance improvements. Access delays of file data 
requests may be classified as direct, which are determined by the cost of accessing the file header or the file data, and indirect, which are the created by contention from other file system requests. Separating control and data addresses the minimization of indirect delays.

The design of MFS gives the ability to match request types to distinct storage structures and storage devices. Each storage structure may be built with different sized disks to increase storage utilization and reduce costs without reducing performance. The small size of control storage allows the use of small mirrored disks to be cost effective. Control storage requires concurrent access, but also has a small storage requirement when compared to data storage. Since the storage capacity of log storage is proportional to the size of main memory caches, small inexpensive disks can be used. The majority of storage capacity would be invested in data storage. Here disks with higher transfer rates and storage density would be used.

Separating control and data storage also simplifies the integration of different storage device technologies into data storage, such as video data stored on read-only disk. Control storage can integrate files stored on different devices into the same name space. The organization of data within that disk is easily modified within control storage, and data from different storage areas could be merged within a single file if desired. Furthermore, different types of data (e.g., text, graphics, audio, video) have different access patterns, and could benefit from different data storage structures. MFS supports multiple storage structures integrated in a single file system.

A major advantage of the structures described for control, data, and log storage is their scalability, not just for space but for response and throughput. The addition of mirrored disks for control storage enhances response and concurrency, which matches the characteristic control request pattern of a high rate of small read/modify/write requests which can be serviced concurrently. The addition of striped disks for data storage enhances throughput (and space of course), which matches the characteristic data request pattern of large sequential data requests.

As for log storage, although the addition of disks certainly enhances response and throughput for cache backup requests by lowering the rate of writes due to compaction, even a single disk helps achieve significant benefits. This is due to our log storage allocation scheme which minimizes disk head movement. Furthermore, if two disks are available, disk heads can be prepositioned so that append-writes, which are the only operations allowed, complete with minimal disk access delays.

Under MFS, the ratio of storage size of control storage to data storage does not differ from more traditional file systems (the same information is stored in both cases). The only additional cost which is required is for log storage, whose size depends on the combined cache sizes, and not the size of control storage or data storage which is where the overwhelming majority of disk space is located. (An effective log storage can be built using two 100MB disks with separate SCSI disk controllers for approximately $\$ 1500$ in today's market.) Adding additional mirrored disks for control storage, is a more significant expense; however, this addition is not a requirement, and provides a performance benefit if added. Overall, these costs lead to improved performance which is significantly higher than traditional file systems, and can be scaled to meet load. As the cost of disks decrease, and the overall size of file systems become larger, MFS becomes more cost-effective.

\section{CONCLUSION}

The motivation behind the MFS design was to improve performance for a broad range of file system workloads. The design is based on distinguishing between control and data requests, and between cache backup and flush requests. These distinctions lead to a distribution of labor which allows its featured storage structures, control, data, and log, aided by caches, to carry out specialized work for which they are well suited. Many of the individual storage structures in isolation have already been carefully researched in prior studies; our contribution is a design which illustrates their effective integration.

In summary, the features of a MFS are the following:

- Multiple isolated storage structures optimized to respond to characteristic access patterns. Isolation avoid delays created by the conflicting modes of disk access.

- Improved bandwidth for large sequential I/O by using disk striping, and improved response time for concurrent accesses of control storage and small files using mirrored disks.

- Lowered access times by reducing the number of disk accesses through caching while maintaining very high reliability through logging.

- Scalable performance to a specified I/O requirement by the addition of disk drives.

- Load balancing across disks due to a natural division of storage by access type (i.e., control and data), and due to the way storage is structured (i.e., mirrored and striped).

\section{ACKNOWLEDGEMENTS}

We thank the reviewers who provided us with constructive comments. We gratefully acknowledge support by DEC, IBM, NCR, NSF, and the Powell Foundation.

\section{References}

[1] Britton, D., and Gray, J., "Disk Shadowing", Proceedings of the 14th Very Large Data Base Conference, September 1988, pp. 331-338.

[2] Cheriton, D. and Finalayson, R., "Log Files: An Extended File Service Exploiting Write-Once Storage.", Proceedings Eleventh Symposium on Operating Systems Principles, November 1987, pp. 139-148. 
[3] Ferrari, D., "Computer Systems Performance Evaluation.", Prentice Hall, 1981.

[4] Floyd, R., and Ellis, C., "Directory Reference Patterns in Hierarchical File Systems", IEEE Transactions on Knowledge and Data Engineering, Volume 1, Number 2, June 1989, pp. 238-247.

[5] Fujitsu M2361A Mini-Disk Drive Customer Engineering Manual.

[6] Gait, J., "The Optical File Cabinet: A RandomAccess File System For Write-Once Optical Disks.", IEEE Computer, Volume 21, Number 6, June 1988, pp. 11-22

[7] Garcia-Molina, H., and Salem, K., "Disk Striping.", International Conference on Data Engineering, February 1986, pp. 336-342.

[8] Hagmann, R., "Reimplementing the Cedar File System Using Logging and Group Commit.", Proceedings Eleventh Symposium on Operating Systems Principles, November 1987, pp. 155-162.

[9] Katz, R., Gibson, G., and Patterson, D., "Disk System Architectures for High Performance Computing." Technical Report, University of California, Berkeley, UCB/CSD 89/497, March 1989, pp. 1-39.

[10] Kim, M., "Synchronized Disk Interleaving.", IEEE Transactions on Computers, Volume C-35, Number 11, November 1986, pp. 978-988.

[11] Lazowska, E. et al., "File Access Performance of Diskless Workstations.", ACM Transaction on Computer Systems, Volume 4, Number 3, August 1986, pp. 238-268.

[12] Livny, M, Khoshafian, S., and Boral, H., "MultiDisk Management Algorithms.", Proceedings Eleventh Symposium on Operating Systems Principles, November 1987, pp. 69-76.

[13] McKusick, M.K, et al., "A Fast File System for UNIX.", Transactions on Computer Systems, Volume 2, Number 3, August 1984, pp. 181-197.

[14] Mullender, S., and Tanenbaum, A., "Immediate Files", Software Practice and Experience, Volume 14, Number 4, April 1984, pp. 365-368.

[15] Nelson, M., "Physical Memory Management in a Network Operating System.", Ph.D. Thesis, University of California, Berkeley, November 1988.

[16] Nelson, M., Welch, B., and Ousterhout, J. "Caching in the Sprite Network File System.", ACM Transactions on Computer Systems, Volume 6, Number 1, February 1988, pp. 134-154.

[17] Ohta, M, and Tezuka, H., "A Fast/tmp File System by Delay Mount Option", USENIX-Summer ' 90 Technical Conference, June 1990, pp. 145-150.

[18] Ousterhout, J., et al., "A Trace Driven Analysis of the Unix 4.2 BSD File System.", Proceedings Tenth Symposium on Operating Systems Principles, December 1985, pp. 15-24.
[19] Ousterhout, J., and Douglis, F., "Beating the I/O Bottleneck: The Case for Log-Structured File Systems.", Operating Systems Review, January 1989, pp. 11-27.

[20] Patterson, D., Gibson, G., and Katz, R., "A Case for Redundant Arrays of Inexpensive Disks (RAID).", ACM SIGMOD 88, June 1988, pp. 109-116.

[21] Powell, M., "The DEMOS File System.", Proceedings Sixth Symposium on Operating Systems Principles, November 1977, pp. 33-42.

[22] Reddy, A., and Banerjee, P., "Performance Evaluation of Multiple-Disk I/O Systems.", 1989 International Conference of Parallel Processing, June 1989, pp. 315-318.

[23] Rosenblum, M, and Ousterhout, J., "The LFS Storage Manager", USENIX-Summer '90 Technical Conference, June 1990, pp. 315-324.

[24] Satyanarayanan, M., "A Study of File Sizes and Functional Lifetimes.", Proceedings Eighth Symposium on Operating Systems Principles, December 1981, pp. 96-108.

[25] Smith, A., "Disk Cache - Miss Ratio Analysis and Design Considerations.",

ACM Transactions on Computer Systems, Volume 3, Number 3, August 1985, pp. 161-203.

[26] Svobodova, L., "A Reliable Object-Oriented Data Repository for a Distributed Computer System.", Proceedings Eighth Symposium on Operating Systems Principles, December 1981, pp. 47-58.

[27] Van Renesse, R., Tanenbaum, A. S., and Wilschut, A., "The Design of a High-Performance File Server", IEEE Transactions on Knowledge and Data Engineering, Volume 1, Number 2, June 1989, pp. 22-27.

[28] Yokoyama, S. and Yamada, S., "A Contiguous High Performance File System", EUUG Spring '89, April 1989, pp. 197-206. 